\title{
BamA and BamD Are Essential for the Secretion of Trimeric Autotransporter Adhesins
}

\section{OPEN ACCESS}

Edited by:

Jai Justin Tree,

University of New South Wales,

Australia

Reviewed by:

Nicholas Noinaj,

Purdue University, United States

Angela Mitchell,

Texas A\&M University, United States

*Correspondence:

Jessica L. Rooke

jessica.rooke@uq.edu.au

lan R. Henderson

I.Henderson@uq.edu.au

i.henderson@imb.uq.edu.au

Specialty section:

This article was submitted to

Microbial Physiology and Metabolism,

a section of the journal

Frontiers in Microbiology

Received: 13 November 2020

Accepted: 26 January 2021

Published: 23 February 2021

Citation:

Rooke JL, Icke C, Wells TJ, Rossiter AE, Browning DF, Morris FC,

Leo JC, Schütz MS, Autenrieth IB,

Cunningham $A F$, Linke $D$ and

Henderson IR (2021) BamA

and BamD Are Essential

for the Secretion of Trimeric

Autotransporter Adhesins.

Front. Microbiol. 12:628879.

doi: 10.3389/fmicb.2021.628879

\section{Jessica L. Rooke1,2*, Christopher Icke1,2, Timothy J. Wells ${ }^{1,3}$, Amanda E. Rossiter ${ }^{2}$, Douglas F. Browning ${ }^{2}$, Faye C. Morris ${ }^{2}$, Jack C. Leo 4,5 , Monika S. Schütz', Ingo B. Autenrieth ${ }^{6}$, Adam F. Cunningham ${ }^{7}$, Dirk Linke ${ }^{5}$ and lan R. Henderson ${ }^{1,2 *}$}

\footnotetext{
${ }^{1}$ Institute for Molecular Bioscience, University of Queensland, St Lucia, QLD, Australia, ${ }^{2}$ Institute of Microbiology and Infection, University of Birmingham, Birmingham, United Kingdom, ${ }^{3}$ The University of Queensland Diamantina Institute, The University of Queensland, Brisbane, QLD, Australia, ${ }^{4}$ Department of Biosciences, Nottingham Trent University, Nottingham, United Kingdom, ${ }^{5}$ Department of Biosciences, University of Oslo, Oslo, Norway, ${ }^{6}$ Institut für Medizinische Mikrobiologie und Hygiene, Universitätsklinikum Tübingen, Tübingen, Germany, ${ }^{7}$ Institute of Immunology

and Immunotherapy, University of Birmingham, Birmingham, United Kingdom
}

The BAM complex in Escherichia coli is composed of five proteins, BamA-E. BamA and BamD are essential for cell viability and are required for the assembly of $\beta$-barrel outer membrane proteins. Consequently, BamA and BamD are indispensable for secretion via the classical autotransporter pathway (Type 5a secretion). In contrast, BamB, BamC, and BamE are not required for the biogenesis of classical autotransporters. Recently, we demonstrated that TamA, a homologue of BamA, and its partner protein TamB, were required for efficient secretion of proteins via the classical autotransporter pathway. The trimeric autotransporters are a subset of the Type 5-secreted proteins. Unlike the classical autotransporters, they are composed of three identical polypeptide chains which must be assembled together to allow secretion of their cognate passenger domains. In contrast to the classical autotransporters, the role of the Bam and Tam complex components in the biogenesis of the trimeric autotransporters has not been investigated fully. Here, using the Salmonella enterica trimeric autotransporter SadA and the structurally similar YadA protein of Yersinia spp., we identify the importance of BamA and BamD in the biogenesis of the trimeric autotransporters and reveal that BamB, BamC, BamE, TamA and TamB are not required for secretion of functional passenger domain on the cell surface.

\section{IMPORTANCE}

The secretion of trimeric autotransporters (TAA's) has yet to be fully understood. Here we show that efficient secretion of TAAs requires the BamA and D proteins, but does not require BamB, C or E. In contrast to classical autotransporter secretion, neither trimeric autotransporter tested required TamA or B proteins to be functionally secreted.

Keywords: autotransporter, trimeric autotransporter adhesin, Bam complex, outer membrane assembly, protein secretion 


\section{INTRODUCTION}

The Type 5 secretion system (T5SS) is the most abundant protein secretion system in Gram-negative bacteria (Henderson and Lam, 2001; Henderson et al., 2004; Desvaux et al., 2005). Recently, the T5SS has been divided into five sub categories; Type $5 \mathrm{a}$ (classical autotransporters), Type 5b (two-partner secretion), Type 5c [trimeric Autotransporter Adhesins (TAA)], Type 5d (fused two-partner) and Type 5e (inverted autotransporters) (Henderson et al., 2000; Tsai et al., 2010; Leo et al., 2012). All sub-classes of the T5SS have the following domains: a signal sequence, secreted passenger domain and a $\beta$-barrel translocation domain. However, the exact composition, order and size of these domains differ between sub-classes (Desvaux et al., 2004; Leo et al., 2012, 2014). In all instances, Type 5 member proteins are often associated with virulence functions, with Type $5 c$ proteins having reported functions such as promoting host cell adherence, biofilm formation, autoaggregation and evasion of complement mediated killing (Leo et al., 2008; Raghunathan et al., 2011; Schindler et al., 2012).

Secretion via the T5SS has mostly been studied in the T5aSS. In this system, the autotransporter protein contains a signal sequence, a passenger domain, and a ca. 300 amino acid $\beta$-barrel translocation domain. The signal sequence directs translocation across the inner membrane via the SecYEG translocon (Desvaux et al., 2006; Hegde and Bernstein, 2006; Desvaux et al., 2007). Once in the periplasm, the signal sequence is cleaved (Knowles et al., 2009). Periplasmic chaperones, such as SurA, Skp, and DegP, assist in the delivery of the remaining protein to the outer membrane $\beta$-barrel Assembly Machinery (Bam) complex (Lazar and Kolter, 1996; Sklar et al., 2007b; Ruiz-Perez et al., 2010). This complex facilitates the insertion of the C-terminal translocation domain into the outer membrane as a monomeric $\beta$-barrel (Knowles et al., 2009). The $\beta$-barrel domain of the T5aSS initiates translocation of the passenger domain to the cell surface and once the passenger domain is located extracellularly it adopts a folded conformation (Leyton et al., 2012). Whilst the precise mechanism of passenger domain translocation is yet to be elucidated, the Bam complex is integrally involved in the secretion process (Jain and Goldberg, 2007; Ieva et al., 2008; Sauri et al., 2009).

In Escherichia coli the Bam complex is comprised of five proteins, BamA-BamE (Voulhoux et al., 2003; Jain and Goldberg, 2007; Rossiter et al., 2011). BamA is an essential outer membrane protein (Werner and Misra, 2005; Knowles et al., 2008). Four periplasmic lipoproteins, BamB-BamE, form a non-covalent interaction with BamA to create the complex (Wu et al., 2005; Knowles et al., 2011). Like BamA, BamD is essential for cell viability (Malinverni et al., 2006). The remaining components are not critical for viability but loss of these components compromises the integrity of the outer membrane and the biogenesis of some outer membrane proteins (Malinverni et al., 2006). Recent studies assessing the contribution of individual BAM complex proteins to the biogenesis of autotransporters have shown that BamA and BamD are essential for the secretion of the classical autotransporters from E. coli (Rossiter et al., 2011). In contrast, BamB, BamC, and BamE were not essential for the secretion or folding of these proteins (Rossiter et al., 2011).
Additionally, we have previously reported that the Translocation Assembly Machinery (Tam) complex was also required for efficient secretion of a T5aSS protein (Selkrig et al., 2012). The Tam complex consists of the integral outer membrane protein TamA and the integral inner membrane protein TamB; TamA shares structural similarity with BamA. Recent studies have demonstrated that the Tam complex facilitates efficient assembly of both Type I fimbriae and Type 5e autotransporters (Heinz et al., 2016; Stubenrauch et al., 2016). However, the precise contribution of the Tam complex to the biogenesis of all members of the T5SS is yet to be elucidated.

In contrast to the $\mathrm{T} 5 \mathrm{aSS}$, the mechanisms of secretion via the other subclasses are less understood. Proteins belonging to the T5cSS, or the Trimeric Autotransporter Adhesin (TAA) family, possess key differences when compared to members of the T5aSS (Linke et al., 2006; Hernandez Alvarez et al., 2008). TAAs are defined by the presence of a short 70-100 amino acid C-terminal translocation domain, and thus the protein must form a homotrimer in order to generate a complete $\beta$-barrel (Cotter et al., 2005; Wells et al., 2007; Wells et al., 2010; Shahid et al., 2012). Consequently, unlike the passenger domains of the T5aSS, which predominantly adopt a monomeric $\beta$-helical conformation, the TAA passenger domains form a trimeric structure composed of oligomeric coiled-coil regions interspersed with distinct head and neck motifs (Linke et al., 2006). The molecular organisation of these proteins presents logistical challenges for the bacterium. For example, the trimer must assemble in a manner that is consistent with the secretion of large passenger domains (up to $5 \mathrm{MDa}$ ) to the bacterial cell surface; at the outer membrane each monomer must be maintained in a conformation that is consistent with pairing to its sister monomers; and monomers must be inserted into the outer membrane whilst being prevented from pairing with the nascent $\beta$-strands of non-partner proteins. Previously, using the archetypical and widely studied TAA protein, YadA from enteropathogenic Yersiniae, we demonstrated that BamA was essential for the assembly of a folded functional trimer into the outer membrane (Lehr et al., 2010). Although the Tam complex has been implicated during the biogenesis of some T5aSS proteins, loss of TamA did not affect the outer membrane accumulation or folding of two TAA proteins (Saragliadis et al., 2018). Due to the complexity of T5cSS, we hypothesised that the other components of the Bam complex and/or the components of the Tam complex would play important roles in co-ordinating the assembly of the TAA homotrimer into the outer membrane. Here we tested this hypothesis by determining the impact that loss of each Bam and Tam component had on the assembly and function of two TAAs, YadA from Yersinia spp., and SadA from Salmonella enterica.

\section{MATERIALS AND METHODS}

\section{Bacterial Strains and Growth Conditions}

The strains and plasmids used in this study are listed in Table 1. All strains were grown in lysogeny broth (LB) with appropriate antibiotics unless otherwise stated. Strains that 
TABLE 1 | Strains and plasmids used in this study

\begin{tabular}{|c|c|c|}
\hline Strain or plasmid & Characteristics & References \\
\hline \multicolumn{3}{|l|}{ Strains } \\
\hline E. coli HB101 & Laboratory E. coli strain & \\
\hline E. coli HB101 bamB:aph & $\begin{array}{l}\text { bamB gene replaced with a } \\
\text { kanamycin cassette }\end{array}$ & $\begin{array}{l}\text { Rossiter et al., } \\
2011\end{array}$ \\
\hline E. coli HB101 bamC:aph & $\begin{array}{l}\text { bamC gene replaced with a } \\
\text { kanamycin cassette }\end{array}$ & $\begin{array}{l}\text { Rossiter et al., } \\
2011\end{array}$ \\
\hline E. coli HB101 bamE:aph & $\begin{array}{l}\text { bamE gene replaced with a } \\
\text { kanamycin cassette }\end{array}$ & $\begin{array}{l}\text { Rossiter et al., } \\
2011\end{array}$ \\
\hline E. coli BW25113 & $\mathrm{K}-12$ derivative & Baba et al., 2006 \\
\hline E. coli BW25113 tamA:aph & $\begin{array}{l}\operatorname{tam} A \text { gene replaced by } \\
\text { kanamycin cassette }\end{array}$ & This study \\
\hline E. coli BW25113 tamB:aph & $\begin{array}{l}\text { tamB gene replaced by } \\
\text { kanamycin cassette }\end{array}$ & This study \\
\hline E. coli JWD3 & $\begin{array}{l}\text { E. coli K-12 BamA } \\
\text { depletion strain. }\end{array}$ & Lehr et al., 2010 \\
\hline E. coli JCM290 & $\begin{array}{l}\text { E. coli K-12 BamD } \\
\text { depletion strain. }\end{array}$ & $\begin{array}{l}\text { Malinverni et al., } \\
2006\end{array}$ \\
\hline \multicolumn{3}{|l|}{ Plasmids } \\
\hline pQE60 Ndel & $\begin{array}{l}\text { pQE60 with added Ndel } \\
\text { restriction site in the } \\
\text { multiple-cloning site and } \\
\text { previously existing } N d e l \text { site } \\
\text { removed }\end{array}$ & $\begin{array}{l}\text { Raghunathan et al., } \\
2011\end{array}$ \\
\hline pDR03 & $\begin{array}{l}\text { pQE60Ndel carrying sadA } \\
\text { gene in the Ndel-Hindlll site }\end{array}$ & $\begin{array}{l}\text { Raghunathan et al., } \\
2011\end{array}$ \\
\hline pET22b(+) & IPTG inducible vector & Invitrogen \\
\hline pET26b(+) & IPTG inducible vector & Invitrogen \\
\hline pDR01 & $\begin{array}{l}\text { sadA gene in the } \\
\text { Ndel-EcoRl sites of } \\
\text { pET22b(+) }\end{array}$ & $\begin{array}{l}\text { Raghunathan et al., } \\
2011\end{array}$ \\
\hline pET26b-SadA & $\begin{array}{l}\text { sadA gene in the } \\
\text { Ndel-EcoRl sites of } \\
\text { pET26b(+) }\end{array}$ & This study \\
\hline plBA2C & $\begin{array}{l}\text { Anhydrotetracycline } \\
\text { inducible vector. }\end{array}$ & IBA Lifesciences \\
\hline plBA2-YadA & $\begin{array}{l}\text { yadA gene in } \\
\text { AHTC-inducible expression } \\
\text { vector pASK-IBA2 }\end{array}$ & $\begin{array}{l}\text { Grosskinsky et al., } \\
2007\end{array}$ \\
\hline pIBA2C-YadA & $\begin{array}{l}\text { yadA gene in the } \mathrm{Xbal}-\mathrm{Sall} \\
\text { sites of pASK-IBA2C }\end{array}$ & This study \\
\hline
\end{tabular}

required antibiotics were supplemented with either $100 \mu \mathrm{g} / \mathrm{ml}$ ampicillin or $50 \mu \mathrm{g} / \mathrm{ml}$ kanamycin. The BamA and BamD depletion strains (JWD3, JCM290) were grown in the presence of $0.05 \%[\mathrm{w} / \mathrm{v}]$ arabinose and $\mathrm{BamA} / \mathrm{BamD}$ were depleted by growth in $0.05 \%$ fructose. Plasmid mediated expression of proteins were induced using a final concentration of $0.5 \mathrm{mM}$ IPTG or $0.2 \mu \mathrm{g} / \mathrm{ml}$ of anhydrotetracycline for T7 and pASK vectors, respectively.

\section{DNA Manipulations and Genetic Techniques}

All plasmids were transformed into the wild-type and mutant strains via heat shock. To construct the pET26b-SadA plasmid, the sadA gene was EcoRI, NdeI digested from pET22b-SadA and religated into a EcoRI, NdeI digested pET26b(+) vector and the correct clone (pET26b-SadA) confirmed by sequencing. The yadA gene was excised from pIBA2-YadA using XbaI and SalI and re-ligated into pASK-IBA2C (IBA Lifesciences) using the same restriction enzymes to create pIBA2C-YadA.

\section{Protein Manipulation Techniques}

Whole cell protein fractions were prepared by centrifuging $1 \mathrm{ml}$ of overnight bacterial culture and resuspending the cell pellet in $2 \times$ Laemmli sample buffer (Sigma) and boiled for $5 \mathrm{~min}$ at $100^{\circ} \mathrm{C}$. Outer membrane protein fractions were prepared as previously described (Parham et al., 2004). Briefly, $50 \mathrm{ml}$ of cultures were grown to an $\mathrm{OD}_{600}$ of 1 . The pellet was resuspended in $20 \mathrm{ml} 10 \mathrm{mM}$ Tris-HCl pH 7.4 buffer and sonicated to lyse cells followed by centrifugation. The supernatant was harvested and centrifuged at $50,000 \times g$. The resulting pellets were washed in $10 \mathrm{mM}$ Tris-HCL pH 7.4, containing $0.2 \%[\mathrm{v} / \mathrm{v}]$ Triton X-100 and subsequently centrifuged at 50,000 $\times g$. The pellets, representing outer membrane fractions, were washed three times in $10 \mathrm{mM}$ Tris- $\mathrm{HCl} \mathrm{pH} 7.4$ before final resuspension in $100 \mu \mathrm{l} 10 \mathrm{mM}$ Tris- $\mathrm{HCl} \mathrm{pH}$ 7.4. Cellular fractions were analysed by SDS-PAGE and stained with Coomassie blue or transferred to a nitrocellulose membrane for Western blotting as described previously (Wells et al., 2009). Western blotting was performed with either a 1:1,000 dilution of anti-SadA antibody, 1:5,000 anti-YadA, anti-BamA, B, C, D, or E antibody or 1:10,000 anti-OmpF. Finally, a 1:10,000 anti-rabbit dilution of IgG alkaline phosphatase was used before detection with NBP-BCIP (nitroblue tetrazolium chloride-5-bromo-4-chloro3 '-indolylphosphate; Sigma-Aldrich) as the substrate.

\section{Aggregation Assay}

Aggregation of the strains was determined using an aggregation settling assay as described previously (Wells et al., 2008). Briefly, overnight cultures were diluted 1:100 and grown in LB until $\mathrm{OD}_{600}$ of 0.8 , at which point anhydrous tetracycline was added. The cultures were grown and standardised to a final $\mathrm{OD}_{600}$ of 1.0 and mixed vigorously for $15 \mathrm{~s}$ prior to the start of the assay. Samples $(150 \mu \mathrm{l})$ were removed at $30 \mathrm{~min}$ intervals taken approximately $0.1 \mathrm{~cm}$ from the liquid surface and transferred into a microtitre plate maintained on ice. At the end of the experiment the $\mathrm{OD}_{600}$ values were measured using a microtitre plate reader.

\section{Biofilm Assay}

The ability of strains to form biofilms was determined using the microtitre plate biofilm assay as described previously (Schembri et al., 2001). Briefly, overnight cultures were inoculated into fresh M9 minimal media to an $\mathrm{OD}_{600 \mathrm{~nm}}$ of 0.02 . The plate was incubated overnight at $37^{\circ} \mathrm{C}$ with shaking. Following incubation, the cultures were removed from the plate and $150 \mu \mathrm{l}$ of $0.1 \%$ $[\mathrm{w} / \mathrm{v}]$ crystal violet stain was added to each well. Upon removal of the stain, the plates were washed with distilled water and left to dry. Addition of an 80:20 ethanol: acetone solution solubilised the crystal violet, and the $\mathrm{OD}_{620} \mathrm{~nm}$ was measured. Each experiment was performed with 12 technical replicates and three biological replicates. Significance was determined by Student's $t$-test. 


\section{Bacterial Adherence to Extracellular Matrix (ECM) Molecules}

Bacterial adherence to ECM molecules was measured via growth essentially as described previously (Wells et al., 2009). Briefly, cells from overnight cultures were grown to $\mathrm{OD}_{600}$ of 1 and appropriately induced for $2 \mathrm{~h}$. These cells were washed in PBS, resuspended to an $\mathrm{OD}_{600}$ of 1 and added to wells coated with $20 \mu \mathrm{g} / \mathrm{ml}$ of collagen I (Sigma-Aldrich). BSA-coated wells (50 $\mu \mathrm{g} / \mathrm{ml}$ ) were used as a control. After $1 \mathrm{~h}$ incubation the wells were washed to remove non-adherent bacteria. Adherent cells were incubated in LB for a further $5 \mathrm{~h}$ prior to enumeration by direct colony counting on agar plates.

\section{Immunofluorescence and Microscopy Imaging}

Fixation and preparation of bacterial cells for immunofluorescence microscopy was performed as previously (Browning et al., 2013). Briefly, fixed cells were put onto polyL-lysine-coated coverslips, washed three times with PBS and then blocked for $1 \mathrm{~h}$ in PBS containing $1 \%[\mathrm{v} / \mathrm{v}]$ bovine serum albumin (Europa Bioproducts). Coverslips were incubated with 1:500 primary antibody for $1 \mathrm{~h}$, washed three times with PBS, and incubated for an additional $1 \mathrm{~h}$ with Alexa Fluor ${ }^{\circledast} 488$ goat anti-rabbit IgG. The coverslips were washed a further three times with sterile PBS before mounting onto glass slides and visualised using either phase contrast or fluorescence using Leica DMRE fluorescence microscope $(100 \times$ objective $)$-DC200 digital camera system.

\section{RESULTS}

\section{BamA and BamD Are Required for the Secretion of SadA}

Previously, we determined that BamA was required for the biogenesis of the trimeric autotransporter YadA (Lehr et al., 2010). To determine if this observation was true for other TAAs, we investigated whether BamA was essential for the biogenesis of a sequence divergent TAA, namely SadA. We first transformed plasmids expressing either SadA (pSadA), YadA (pYadA) and corresponding empty vector(-) into E. coli HB101. Outer membrane fractions of induced cells were probed for either SadA or YadA using specific antibodies. SadA and YadA were detected in the outer membrane of HB101, demonstrating specificity of the antibodies used (Figures 1A,B). To determine the role of BamA during SadA outer membrane biogenesis, pSadA was transformed into the BamA depletion strain E. coli JWD3 (Lehr et al., 2010). After growth in the presence or absence of arabinose, outer membrane fractions were harvested and SadA was detected by Western immunoblotting. SadA could be detected under BamA replete conditions (Figure 1C). In contrast, like BamA and the BamA-dependent protein OmpF, SadA levels were severely diminished after growth in BamAdepleted cells.

BamD is the second component of the Bam complex that is vital for cell viability (Malinverni et al., 2006). To determine whether BamD is required for TAA secretion we followed a similar method to BamA above. The secretion of SadA to the outer membrane was investigated in a BamD depletion strain (JCM290). When BamD was depleted from the cell, the protein levels of SadA and $\mathrm{OmpF} / \mathrm{C}$ were considerably depleted in the outer membrane (Figure 1D), which was similar to the results observed for BamA.

To further confirm the observations that the essential Bam components are required for the secretion of TAAs, YadA was investigated for secretion under BamA and D depletion conditions. JWD3 and JCM290 were transformed pYadA. After growth in the absence or presence of arabinose, the outer membrane proteins were isolated as previously described. The proteins were analysed by SDS-PAGE and Western immunoblot. Under BamA replete conditions, YadA was detected in the outer membrane of JWD3. Upon depletion of BamA, YadA was no longer detectable in the outer membrane (Figure 1E), as previously reported (Lehr et al., 2010). Similarly, when BamD was depleted from the cells, YadA could no longer be detected in the outer membrane (Figure 1F). These data reinforce previous observations that BamA is required for the biogenesis of TAAs and demonstrate for the first time that $\mathrm{BamD}$ is essential for TAA biogenesis.

\section{The Non-essential Bam Components Are Not Required for Outer Membrane Localisation of TAA's}

While BamA and BamD are required for the biogenesis of TAAs, we sought to investigate the contribution of the nonessential components of the Bam complex (BamB, C, and E) for TAA biogenesis. E. coli wild-type and bamB, bamC, and bamE deletion mutants (Rossiter et al., 2011) were transformed with pDR03 $(\mathrm{SadA}+)$ or pIBA2-YadA (YadA + ) or empty vector. Outer membrane protein fractions of each sample were probed for the presence of SadA, YadA and each Bam component. The Bam mutants were confirmed by Western blot analysis (Figure 2A) and strains expressing either SadA or YadA were shown to have the protein present in the outer membrane fractions (Figure 2B). The absence of BamB, BamC, or BamE did not affect the accumulation of either YadA or SadA in the outer membrane (Figure 2B).

\section{The Tam Complex Is Not Essential for Outer Membrane Localisation of TAA's}

Earlier studies demonstrated that the Tam complex is required for the correct biogenesis of members of the T5aSS. Therefore, we hypothesised that the TAAs proteins would also require the assistance of the Tam complex for biogenesis. To test this, we first created $\operatorname{tam} A$ and $\operatorname{tam} B$ null mutants by P1 transducing the corresponding mutations from the Keio collection, and confirmed by Western blot analysis of whole cell protein fractions (Figure 3A). The resulting mutants were transformed with either the empty vector or plasmids encoding either SadA or YadA and outer membrane fractions probed for presence of YadA or SadA (Figure 3B). In the absence of TamA or TamB, both YadA and SadA 

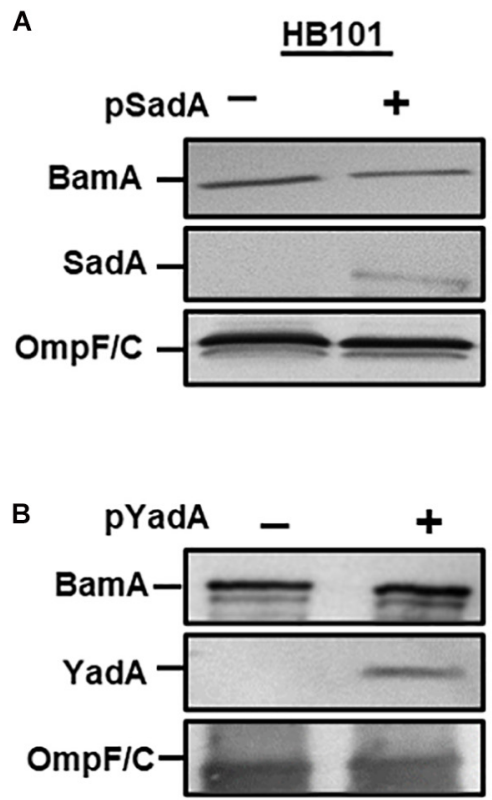
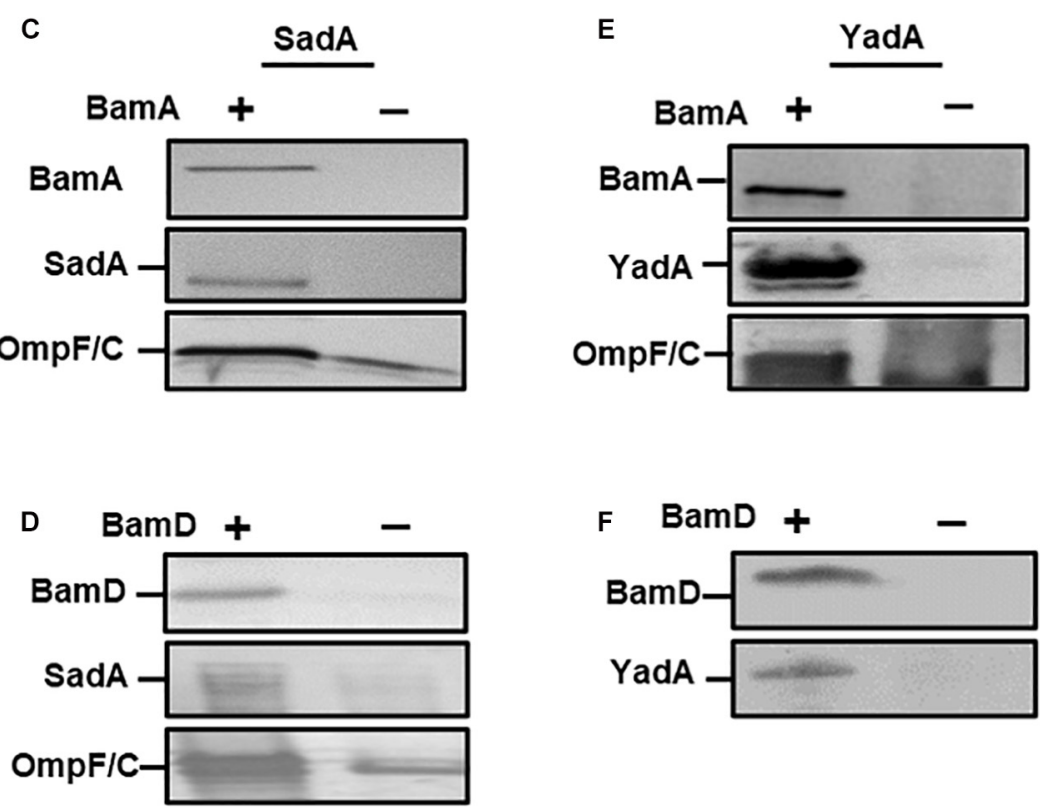

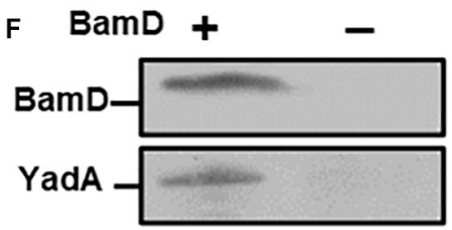

FIGURE 1 | BamA and BamD are required for secretion of SadA. Western blot analysis of outer membrane fractions of HB101 with either empty vector (-), pSadA (A) or pYadA (B) plasmids (+). Similar analysis of (C) BamA depletion JWD3 strain and (D) BamD depletion strain (JCM290)( \pm depletion) with SadA + plasmids Western blot analysis of outer membrane fractions of (E) BamA and (F) BamD depletion strains( \pm depletion) with YadA plasmid. Fractions were probed with either anti-BamA, anti-BamD, anti-SadA, anti-YadA, and anti-OmpF/C antisera. Bands indicate the presence of the protein in the outer membrane.
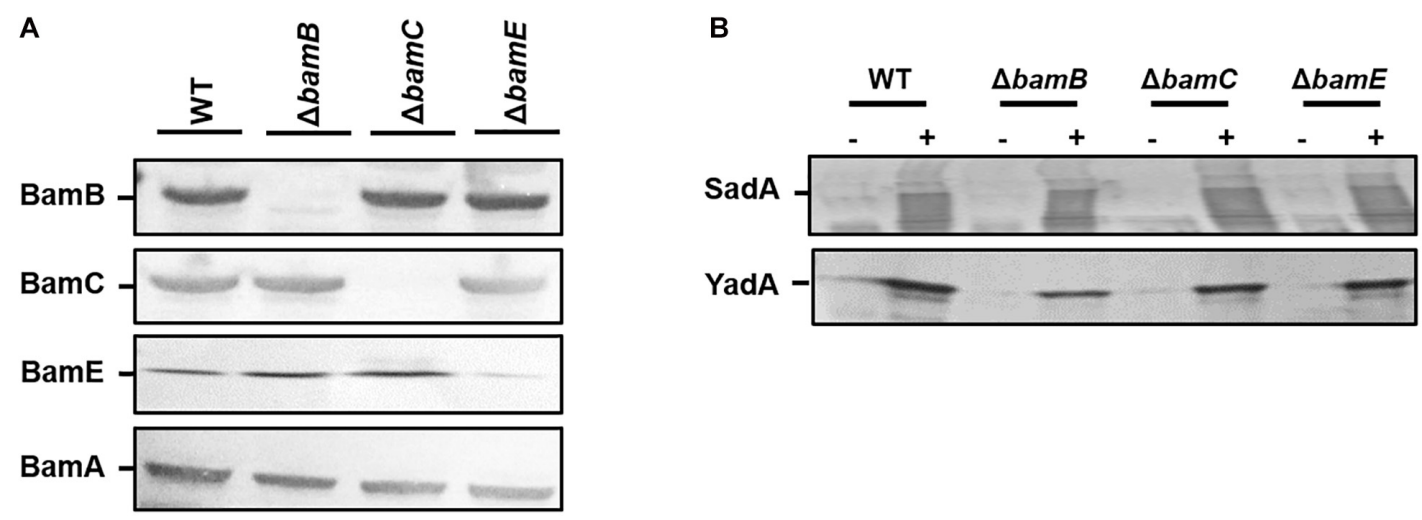

FIGURE 2 | Non-essential BAM components are not required for outer membrane localisation of trimeric autotransporters. (A) Western blot analysis of wild-type HB101 and bamB, C or E mutant strains confirming the loss of each protein in the corresponding mutant. Outer membrane fractions were probed with either anti-BamA, B, C, E specific antibodies. (B) Outer membrane fractions of wild-type and bam mutant strains expressing either an empty vector control (-) or SadAYYada (+) analysis by Western blot. Protein fractions were probed using either anti-YadA or anti-SadA specific antibodies confirming the presence of the proteins in the outer membrane.

are incorporated into the outer membrane at levels similar to the wild-type parental strain, suggesting that the Tam complex is not essential for the localisation of TAAs to the OM (Figure 3B).

\section{TAA Passenger Domains Are Secreted to the Cell Surface in bam and tam Mutants}

Previously, we observed intermediates of the T5aSS that are localised to the outer membrane but are unable to translocate their cognate passenger domains to the cell surface (Leyton et al., 2011). Therefore, we hypothesised that whilst the non-essential Bam components and the Tam components were not required for localisation to the outer membrane, they might be responsible for holding the C-terminal translocation unit and/or passenger domains in a conformation that permits secretion of the passenger domain to the cell surface. The surface exposure of both SadA and YadA were determined by specific immunofluorescence microscopy in WT, BamB, C, E and TamA and B mutants (Figure 4). Both YadA and SadA 


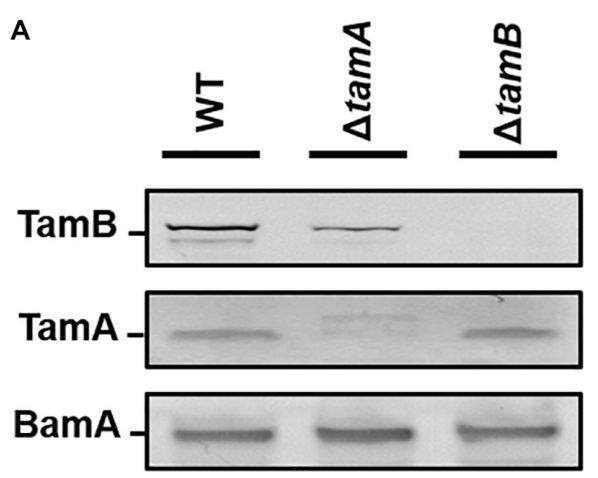

B

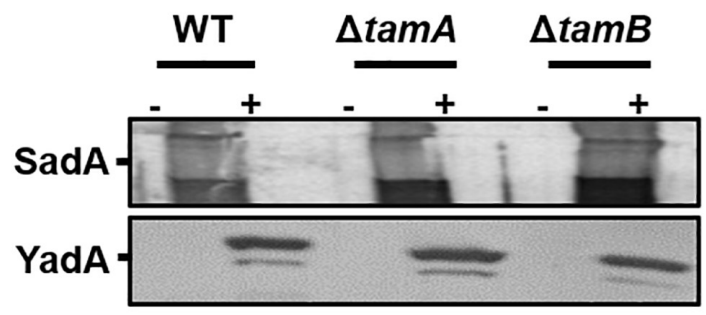

FIGURE 3 | TamA and TamB are not required for outer membrane insertion of trimeric autotransporters. (A) Western blot analysis of wild-type HB101 and tamA and $\operatorname{tam} B$ mutant outer membrane fractions confirming the loss of each protein in the corresponding mutant using antibodies specific to TamA and B with anti-BamA specific antibodies used as a positive control. (B) Western blot analysis of wild-type HB101 and tamA and tamB mutant outer membrane fractions expressing either empty vector (-) or YadA/SadA (+) using YadA or SadA specific antibodies to confirm the presence of the proteins in the outer membrane.

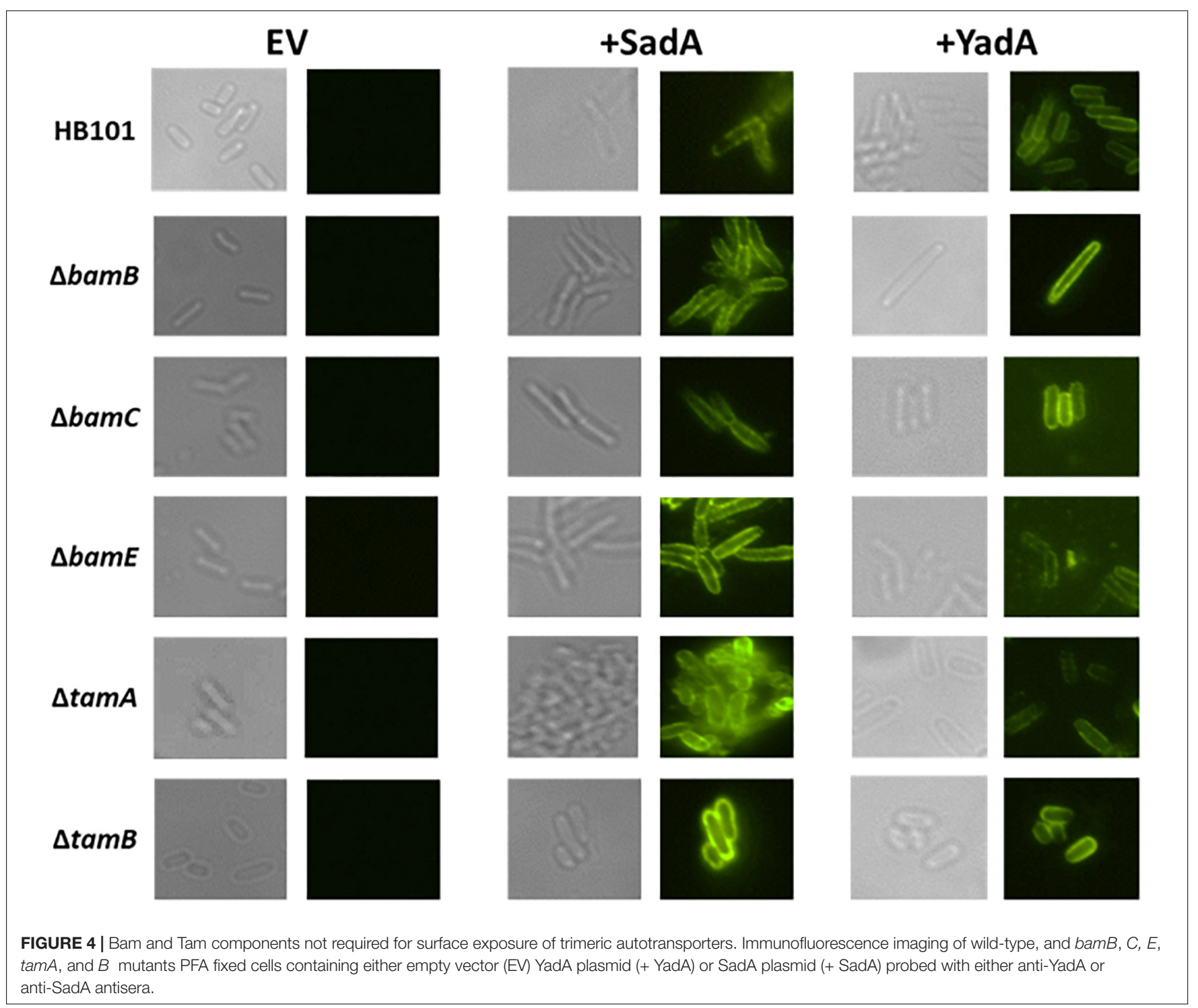



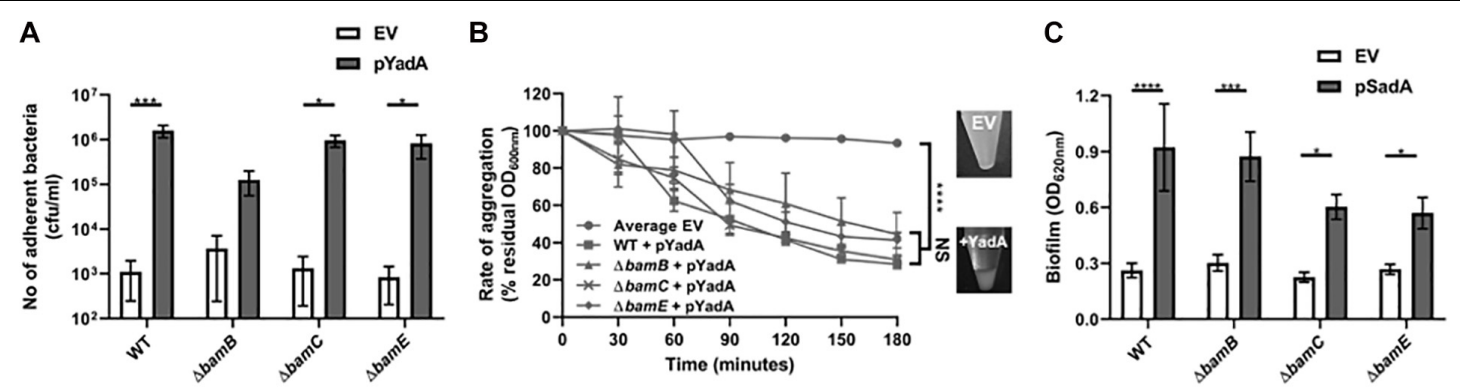

FIGURE 5 | Trimeric autotransporters are functional in the absence of the non-essential Bam components. (A) Collagen I binding by wild-type and Bam mutant HB101 strains containing either EV or YadA + plasmid. Adhesion was examined via measuring growth in microtitre plates after binding and washing. Significance $(p<0.05)$ was determined by a Student's $t$-test. Error bars represent standard error of the mean (SEM) of at least 8 technical replicates and 3 separate experiments (B) Aggregation of wild-type and mutant HB101 strains containing either EV (empty vector) or YadA + plasmid. The pellet formed by autoaggregation can be visibly compared after $3 \mathrm{~h}$ (insets). Significance $(p<0.05)$ was determined by a 2-way Anova with Turkey's correction for multiple comparisons. Error bars represent SEM of 3 separate experiments. (C) Biofilm formation by wild-type and mutant HB101 strains containing either EV or SadA expressing plasmid. Biofilm formation was examined in polystyrene microtitre plates. Significance $(p<0.05)$ was determined by a Student's $t$-test Error bars represent standard deviation of at least 12 technical replicates and 5 separate experiments. Significance was represented as follows: ${ }^{\star} p<0.05,{ }^{\star \star \star} p<0.001$, and ${ }^{\star \star \star \star} p<0.0001$.

were detectable on the surface of all strains containing the corresponding vector including all three Bam mutants and both Tam mutants (Figure 4).

\section{TAA Passenger Domains Are Functional in bam and tam Mutants}

Although the Tam and the non-essential Bam components are not required for surface localisation of the passenger domain, they may be required for the correct folding and function of TAA passenger domains on the cell surface. Thus, we investigated whether the TAA proteins retained functional competence in these mutant backgrounds. As YadA is known to mediate binding to collagen I and to promote autoaggregation (Nummelin et al., 2004), the E. coli bamB, bamC, and bamE mutants expressing $\operatorname{yad} A$ were tested in a growth-based collagen binding assay. Expression of yadA in WT and bamC and $E$ mutant strains promoted a significant increase in binding to collagen I compared to the empty vector controls (Figure 5A). Whilst there was an increase in bacterial adherence to collagen I in the bamB mutant, this was not significantly different to the empty vector control (Figure 5A). As bamB mutants are known to have a reduced growth rate, we concluded that this lack of significance was probably due to slower growth of the bamB mutant. YadA is also known to promote cell-cell autoaggregation therefore we tested the ability of YadA to promote autoaggregation in the non-essential Bam mutants. Induction of yadA expression in all strains led to significant autoaggregation, in contrast to empty vector control strains which had no discernible aggregation (Figure 5B). Previously, we demonstrated that SadA could promote biofilm production in vitro (Raghunathan et al., 2011). Thus, we investigated the ability of wild-type and Bam mutant strains, either expressing or not expressing SadA, for their ability to form a biofilm on polystyrene plates when grown in M9 minimal medium. All strains expressing SadA had significantly $(P<0.05)$ higher biofilm formation than the empty vector controls (Figure 5C).

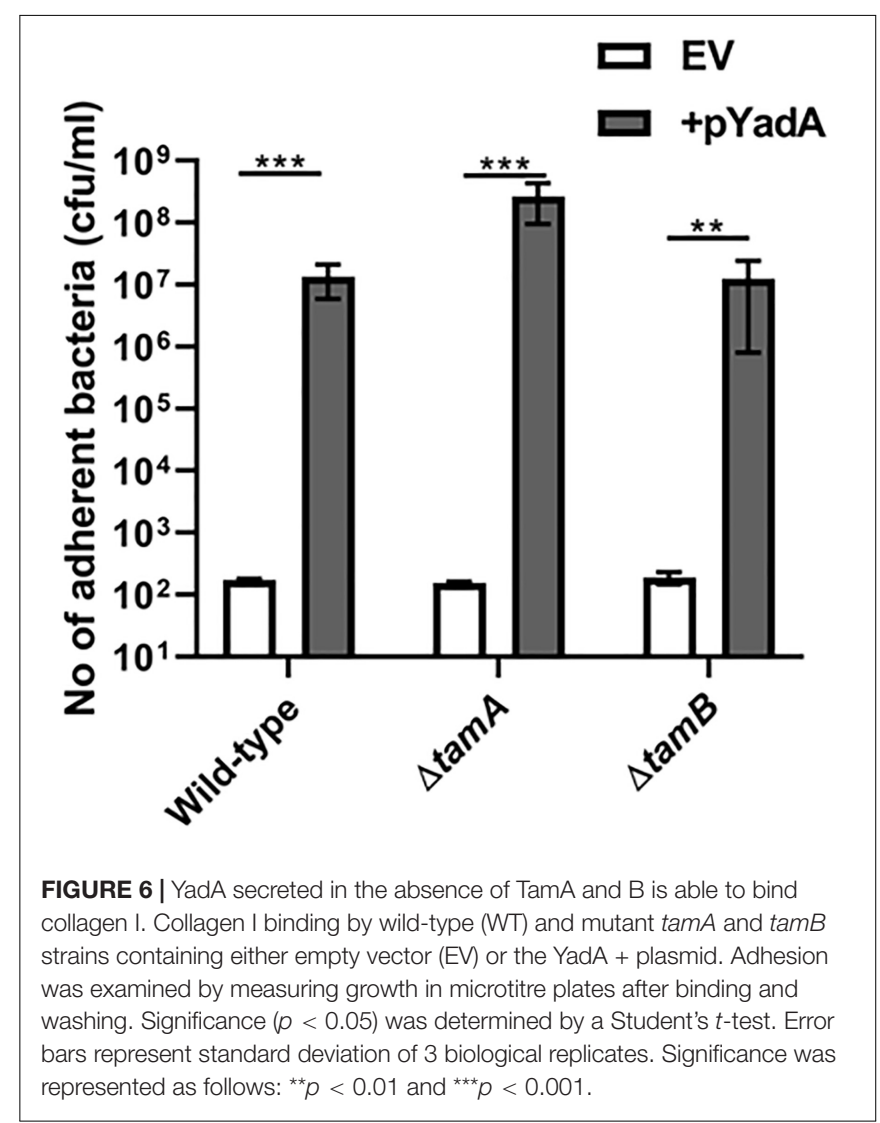

To investigate whether TamA and TamB were required for correct folding of TAA passenger domains, the tam mutants described above were monitored for SadA and YadA function. As above we tested the WT and $\operatorname{tam} A$ and $B$ mutants for their ability to bind collagen I. All three strains expressing YadA had significantly higher collagen I binding compared to empty vector controls (Figure 6). We found that $\operatorname{tam} A$ and $B$ 
mutants in E. coli form high biofilm (data not shown) and thus were unable to determine whether SadA could promote biofilm formation in these strains. These data suggest that TAAs are secreted, folded and function even in the absence of BamB, C, E, TamA or B mutants.

\section{DISCUSSION}

The proteins SadA and YadA are both trimeric autotransporter adhesins, however they vary greatly in sequence homology, length, and function. YadA is the prototypical TAA, its trimeric structure composed of a $\beta$-barrel in the outer membrane, with a single stalk, neck and head domain extending $35 \mathrm{~nm}$ into the extracellular environment (Koretke et al., 2006). In contrast, SadA extends $108 \mathrm{~nm}$ and is composed of multiple stalk, neck and head domains alternatingly dispersed along the length of the passenger domain (Hartmann et al., 2012). Here we found that both BamA and $\mathrm{BamD}$ are required for the secretion and function of YadA and SadA, and by implication all TAAs. This is in agreement with studies on other outer membrane proteins (Rossiter et al., 2011), and is further evidence that BamA and BamD are essential to assemble proteins into the outer membrane (Malinverni et al., 2006; Hagan et al., 2010). In contrast, both SadA and YadA were functionally assembled in the outer membrane in $\operatorname{bamB}, \operatorname{bam} C$, and bamE mutant strains. These three lipoproteins have been shown to be non-essential in E. coli and although they are needed for the secretion of some outer membrane proteins (Charlson et al., 2006; Hagan et al., 2010), are also not essential for others (Sklar et al., 2007a; Volokhina et al., 2009; Weirich et al., 2017). Importantly, our findings corroborate results from Yersinia enterocolitica, that showed the non-essential Bam components are not required for YadA secretion or function, suggesting our results using heterologous expression in $E$. coli is representative of native outer membrane biogenesis (Weirich et al., 2017). In addition, and consistent with previous studies (Saragliadis et al., 2018), we observe that TamA and B are not required for the biogenesis or function of TAA proteins. However, TamA and B have been implicated in the secretion of classical autotransporters (Selkrig et al., 2012), suggesting TamA and B may be involved in secretion of a specific subset of Type 5 proteins or may have importance under specific conditions.

Recent evidence suggests that trimerisation of TAA's occurs in the periplasm prior to outer membrane insertion (Sikdar and Bernstein, 2019) and that TAA passenger translocation occurs via hairpin intermediates (Chauhan et al., 2019). The ability of SadA to induce biofilm formation and YadA to induce autoaggregation in all of the mutants at levels similar to the wild-type suggests that these proteins are functionally assembled in a folded state on the surface of the bacterium. These data indicate that the non-essential Bam components and the Tam components are not essential for the translocation or folding of TAAs on the bacterial cell surface. This is in agreement with other studies that demonstrate other proteins such as Pet, TolC are functionally assembled in bamB mutants (Charlson et al., 2006; Rossiter et al., 2011). Additionally, TAA proteins such as NhhA exist in bacterial species (Neisseria meningitidis) that do not have a BamB homologue, suggesting it is not required for proper assembly of this protein class (Volokhina et al., 2009). Interestingly, although SadA protein levels in outer membrane preparations were similar in all strains, biofilm formation induced by SadA was significantly lower in a bamC and bamE knockout compared to wild-type $(p<0.05)$ (Figure 5C). This suggests that these lipoproteins may play a more subtle role in TAA secretion, and multiple lipoprotein knockouts may lead to secretion defects. Indeed, in $N$. meningitidis a bamC/E double knockout leads to a lethal phenotype (Volokhina et al., 2009) and it has recently been shown that a double bamB/bamE mutant is lethal in E. coli (Tellez and Misra, 2012), suggesting the non-essential lipoproteins have some redundancy.

In conclusion, we show that the non-essential components of the Bam complex and both TamA and B are not essential for TAA assembly. Given the complex nature of TAA biogenesis, due to large and complex passenger structures and the need for trimerisation, it is plausible that loss of Bam and/or Tam proteins could lead to a reduced efficiency of TAA passenger translocation. Further experimental evidence would be required to investigate this hypothesis.

\section{DATA AVAILABILITY STATEMENT}

The raw data supporting the conclusions of this article will be made available by the authors, without undue reservation.

\section{AUTHOR CONTRIBUTIONS}

JR: conceptualisation, data curation, formal analysis, investigation, methodology, project administration, and writing-original draft. CI: conceptualisation, data curation, formal analysis, investigation, methodology, and writingreview and editing. TW: formal analysis, investigation, methodology, resources, supervision, writing-original draft, and writing-review and editing. AR: data curation, formal analysis, investigation, methodology, project administration, resources, and writing - review and editing. DB: data curation, formal analysis, investigation, project administration, resources, supervision, and writing-review and editing. FM: conceptualisation, investigation, methodology, project administration, resources, validation, and writing-review and editing. JL, MS, and DL: methodology, and resources, writing-review and editing. AC: formal analysis, funding acquisition, investigation, methodology, supervision, and writing-review and editing. IH: conceptualisation, formal analysis, funding acquisition, investigation, methodology, project administration, resources, supervision, and writingreview and editing. All authors contributed to the article and approved the submitted version.

\section{FUNDING}

This work was supported by Research Council of Norway Young Investigator grant (no. 249793 to JL). 


\section{REFERENCES}

Henderson, I. R., Navarro-Garcia, F., Desvaux, M., Fernandez, R. C., and Ala'Aldeen, D. (2004). Type V protein secretion pathway: the autotransporter story. Microbiol. Mol. Biol. Rev. 68, 692-744. doi: 10.1128/mmbr.68.4.692-744. 2004

Desvaux, M., Khan, A., Beatson, S. A., Scott-Tucker, A., and Henderson, I. R. (2005). Protein secretion systems in Fusobacterium nucleatum: genomic identification of Type 4 piliation and complete Type $\mathrm{V}$ pathways brings new insight into mechanisms of pathogenesis. Biochim. Biophys. Acta 1713, 92-112. doi: 10.1016/j.bbamem.2005.05.002

Henderson, I. R., and Lam, A. C. (2001). Polymorphic proteins of Chlamydia spp.-autotransporters beyond the Proteobacteria. Trends Microbiol. 9, 573-578. doi: $10.1016 /$ s0966-842x(01)02234-x

Henderson, I. R., Cappello, R., and Nataro, J. P. (2000). Autotransporter proteins, evolution and redefining protein secretion. Trends Microbiol. 8, 529-532. doi: 10.1016/s0966-842x(00)01853-9

Leo, J. C., Grin, I., and Linke, D. (2012). Type V secretion: mechanism(s) of autotransport through the bacterial outer membrane. Philos. Trans. R. Soc. Lond. B Biol. Sci. 367, 1088-1101. doi: 10.1098/rstb.2011.0208

Tsai, J. C., Yen, M. R., Castillo, R., Leyton, D. L., Henderson, I. R., and Saier, M. H. Jr. (2010). The bacterial intimins and invasins: a large and novel family of secreted proteins. PLoS One 5:e14403. doi: 10.1371/journal.pone.0014403

Desvaux, M., Parham, N. J., and Henderson, I. R. (2004). The autotransporter secretion system. Res. Microbiol. 155, 53-60. doi: 10.1016/j.resmic.2003.10.002

Leo, J. C., Oberhettinger, P., Schutz, M., and Linke, D. (2014). The inverse autotransporter family: intimin, invasin and related proteins. Int. J. Med. Microbiol. 305, 276-282. doi: 10.1016/j.ijmm.2014.12.011

Raghunathan, D., Wells, T. J., Morris, F. C., Shaw, R. K., Bobat, S., Peters, S. E., et al. (2011). SadA, a trimeric autotransporter from Salmonella enterica serovar Typhimurium, can promote biofilm formation and provides limited protection against infection. Infect. Immun. 79, 4342-4352. doi: 10.1128/iai.05592-11

Leo, J. C., Elovaara, H., Brodsky, B., Skurnik, M., and Goldman, A. (2008). The Yersinia adhesin YadA binds to a collagenous triple-helical conformation but without sequence specificity. Protein Eng. Des. Sel. 21, 475-484. doi: 10.1093/ protein/gzn025

Schindler, M. K., Schutz, M. S., Muhlenkamp, M. C., Rooijakkers, S. H., Hallstrom, T., Zipfel, P. F., et al. (2012). Yersinia enterocolitica YadA mediates complement evasion by recruitment and inactivation of C3 products. J. Immunol. 189, 4900-4908. doi: 10.4049/jimmunol.1201383

Hegde, R. S., and Bernstein, H. D. (2006). The surprising complexity of signal sequences. Trends Biochem. Sci. 31, 563-571. doi: 10.1016/j.tibs.2006.08.004

Desvaux, M., Scott-Tucker, A., Turner, S. M., Cooper, L. M., Huber, D., Nataro, J. P., et al. (2007). A conserved extended signal peptide region directs posttranslational protein translocation via a novel mechanism. Microbiology 153, 59-70. doi: 10.1099/mic.0.29091-0

Desvaux, M., Cooper, L. M., Filenko, N. A., Scott-Tucker, A., Turner, S. M., Cole, J. A., et al. (2006). The unusual extended signal peptide region of the type $\mathrm{V}$ secretion system is phylogenetically restricted. FEMS Microbiol. Lett. 264, 22-30. doi: 10.1111/j.1574-6968.2006.00425.x

Knowles, T. J., Scott-Tucker, A., Overduin, M., and Henderson, I. R. (2009). Membrane protein architects: the role of the BAM complex in outer membrane protein assembly. Nat. Rev. Microbiol. 7, 206-214. doi: 10.1038/nrmicro 2069

Lazar, S. W., and Kolter, R. (1996). SurA assists the folding of Escherichia coli outer membrane proteins. J. Bacteriol. 178, 1770-1773. doi: 10.1128/jb.178.6.17701773.1996

Sklar, J. G., Wu, T., Kahne, D., and Silhavy, T. J. (2007b). Defining the roles of the periplasmic chaperones SurA, Skp, and DegP in Escherichia coli. Genes Dev. 21, 2473-2484. doi: 10.1101/gad.1581007

Ruiz-Perez, F., Henderson, I. R., and Nataro, J. P. (2010). Interaction of FkpA, a peptidyl-prolyl cis/trans isomerase with EspP autotransporter protein. Gut Microbes 1, 339-344. doi: 10.4161/gmic.1.5.13436

Leyton, D. L., Rossiter, A. E., and Henderson, I. R. (2012). From self sufficiency to dependence: mechanisms and factors important for autotransporter biogenesis. Nat. Rev. Microbiol. 10, 213-225. doi: 10.1038/nrmicro2733

Ieva, R., Skillman, K. M., and Bernstein, H. D. (2008). Incorporation of a polypeptide segment into the beta-domain pore during the assembly of a bacterial autotransporter. Mol. Microbiol. 67, 188-201. doi: 10.1111/j.13652958.2007.06048.x

Jain, S., and Goldberg, M. B. (2007). Requirement for YaeT in the outer membrane assembly of autotransporter proteins. J. Bacteriol. 189, 5393-5398. doi: 10.1128/ jb.00228-07

Sauri, A., Soprova, Z., Wickstrom, D., de Gier, J. W., Van der Schors, R. C., Smit, A. B., et al. (2009). The Bam (Omp85) complex is involved in secretion of the autotransporter haemoglobin protease. Microbiology 155, 3982-3991. doi: 10.1099/mic.0.034991-0

Rossiter, A. E., Leyton, D. L., Tveen-Jensen, K., Browning, D. F., Sevastsyanovich, Y., Knowles, T. J., et al. (2011). The essential beta-barrel assembly machinery complex components $\mathrm{BamD}$ and BamA are required for autotransporter biogenesis. J. Bacteriol. 193, 4250-4253. doi: 10.1128/jb.00192-11

Voulhoux, R., Bos, M. P., Geurtsen, J., Mols, M., and Tommassen, J. (2003). Role of a highly conserved bacterial protein in outer membrane protein assembly. Science 299, 262-265. doi: 10.1126/science.1078973

Knowles, T. J., Jeeves, M., Bobat, S., Dancea, F., McClelland, D., Palmer, T., et al. (2008). Fold and function of polypeptide transport-associated domains responsible for delivering unfolded proteins to membranes. Mol. Microbiol. 68, 1216-1227. doi: 10.1111/j.1365-2958.2008.06225.x

Werner, J., and Misra, R. (2005). YaeT (Omp85) affects the assembly of lipiddependent and lipid-independent outer membrane proteins of Escherichia coli. Mol. Microbiol. 57, 1450-1459. doi: 10.1111/j.1365-2958.2005.04775.x

Knowles, T. J., Browning, D. F., Jeeves, M., Maderbocus, R., Rajesh, S., Sridhar, P., et al. (2011). Structure and function of BamE within the outer membrane and the beta-barrel assembly machine. EMBO Rep. 12, 123-128. doi: 10.1038/ embor.2010.202

Wu, T., Malinverni, J., Ruiz, N., Kim, S., Silhavy, T. J., and Kahne, D. (2005). Identification of a multicomponent complex required for outer membrane biogenesis in Escherichia coli. Cell 121, 235-245. doi: 10.1016/j.cell.2005.02.015

Malinverni, J. C., Werner, J., Kim, S., Sklar, J. G., Kahne, D., Misra, R., et al. (2006). YfiO stabilizes the YaeT complex and is essential for outer membrane protein assembly in Escherichia coli. Mol. Microbiol. 61, 151-164. doi: 10.1111/j.13652958.2006.05211.x

Selkrig, J., Mosbahi, K., Webb, C. T., Belousoff, M. J., Perry, A. J., Wells, T. J., et al. (2012). Discovery of an archetypal protein transport system in bacterial outer membranes. Nat. Struct. Mol. Biol. 19, 506-510,S1.

Stubenrauch, C., Belousoff, M. J., Hay, I. D., Shen, H. H., Lillington, J., Tuck, K. L., et al. (2016). Effective assembly of fimbriae in Escherichia coli depends on the translocation assembly module nanomachine. Nat. Microbiol. 1:16064.

Heinz, E., Stubenrauch, C. J., Grinter, R., Croft, N. P., Purcell, A. W., Strugnell, R. A., et al. (2016). Conserved Features in the Structure, Mechanism, and Biogenesis of the Inverse Autotransporter Protein Family. Genome Biol. Evol. 8, 1690-1705. doi: 10.1093/gbe/evw112

Hernandez Alvarez, B., Hartmann, M. D., Albrecht, R., Lupas, A. N., Zeth, K., and Linke, D. (2008). A new expression system for protein crystallization using trimeric coiled-coil adaptors. Protein Eng. Des. Sel. 21, 11-18. doi: 10.1093/ protein/gzm071

Linke, D., Riess, T., Autenrieth, I. B., Lupas, A., and Kempf, V. A. (2006). Trimeric autotransporter adhesins: variable structure, common function. Trends Microbiol. 14, 264-270. doi: 10.1016/j.tim.2006.04.005

Wells, T. J., Totsika, M., and Schembri, M. A. (2010). Autotransporters of Escherichia coli: a sequence-based characterization. Microbiology 156, 24592469. doi: 10.1099/mic.0.039024-0

Wells, T. J., Tree, J. J., Ulett, G. C., and Schembri, M. A. (2007). Autotransporter proteins: novel targets at the bacterial cell surface. FEMS Microbiol. Lett. 274, 163-172. doi: 10.1111/j.1574-6968.2007.00833.x

Cotter, S. E., Surana, N. K., and St Geme, J. W. III (2005). Trimeric autotransporters: a distinct subfamily of autotransporter proteins. Trends Microbiol. 13, 199-205. doi: 10.1016/j.tim.2005.03.004

Shahid, S. A., Bardiaux, B., Franks, W. T., Krabben, L., Habeck, M., van Rossum, B. J., et al. (2012). Membrane-protein structure determination by solid-state NMR spectroscopy of microcrystals. Nat. Methods 9, 1212-1217.

Lehr, U., Schutz, M., Oberhettinger, P., Ruiz-Perez, F., Donald, J. W., Palmer, T., et al. (2010). C-terminal amino acid residues of the trimeric autotransporter adhesin YadA of Yersinia enterocolitica are decisive for its recognition and assembly by BamA. Mol. Microbiol. 78, 932-946. doi: 10.1111/j.1365-2958. 2010.07377.x 
Saragliadis, A., Trunk, T., and Leo, J. C. (2018). Producing gene deletions in Escherichia coli by $\mathrm{P} 1$ transduction with excisable antibiotic resistance cassettes. J. Vis. Exp. 139:58267. doi: 10.3791/58267

Parham, N. J., Srinivasan, U., Desvaux, M., Foxman, B., Marrs, C. F., and Henderson, I. R. (2004). PicU, a second serine protease autotransporter of uropathogenic Escherichia coli. FEMS Microbiol. Lett. 230, 73-83. doi: 10.1016/ s0378-1097(03)00862-0

Wells, T. J., McNeilly, T. N., Totsika, M., Mahajan, A., Gally, D. L., and Schembri, M. A. (2009). The Escherichia coli O157:H7 EhaB autotransporter protein binds to laminin and collagen I and induces a serum IgA response in O157:H7 challenged cattle. Environ. Microbiol. 11, 1803-1814. doi: 10.1111/j.1462-2920. 2009.01905.x

Wells, T. J., Sherlock, O., Rivas, L., Mahajan, A., Beatson, S. A., Torpdahl, M., et al. (2008). EhaA is a novel autotransporter protein of enterohemorrhagic Escherichia coli O157:H7 that contributes to adhesion and biofilm formation. Environ. Microbiol. 10, 589-604. doi: 10.1111/j.1462-2920.2007. 01479.x

Schembri, M. A., Kjaergaard, K., Sokurenko, E. V., and Klemm, P. (2001). Molecular characterization of the Escherichia coli FimH adhesin. J. Infect. Dis. 183(Suppl. 1), S28-S31.

Browning, D. F., Matthews, S. A., Rossiter, A. E., Sevastsyanovich, Y. R., Jeeves, M., Mason, J. L., et al. (2013). Mutational and topological analysis of the Escherichia coli BamA protein. PLoS One 8:e84512. doi: 10.1371/journal.pone.008 4512

Leyton, D. L., Sevastsyanovich, Y. R., Browning, D. F., Rossiter, A. E., Wells, T. J., Fitzpatrick, R. E., et al. (2011). Size and conformation limits to secretion of disulfide-bonded loops in autotransporter proteins. J. Biol. Chem. 286, 42283-42291. doi: 10.1074/jbc.m111.306118

Nummelin, H., Merckel, M. C., Leo, J. C., Lankinen, H., Skurnik, M., and Goldman, A. (2004). The Yersinia adhesin YadA collagen-binding domain structure is a novel left-handed parallel beta-roll. EMBO J. 23, 701-711. doi: 10.1038/sj. emboj.7600100

Koretke, K. K., Szczesny, P., Gruber, M., and Lupas, A. N. (2006). Model structure of the prototypical non-fimbrial adhesin YadA of Yersinia enterocolitica. J. Struct. Biol. 155, 154-161. doi: 10.1016/j.jsb.2006. 03.012

Hartmann, M. D., Grin, I., Dunin-Horkawicz, S., Deiss, S., Linke, D., Lupas, A. N., et al. (2012). Complete fiber structures of complex trimeric autotransporter adhesins conserved in enterobacteria. Proc. Natl. Acad. Sci. U.S.A. 109, 2090720912. doi: 10.1073/pnas.1211872110

Hagan, C. L., Kim, S., and Kahne, D. (2010). Reconstitution of outer membrane protein assembly from purified components. Science 328, 890-892. doi: 10. 1126/science.1188919
Charlson, E. S., Werner, J. N., and Misra, R. (2006). Differential effects of yfgL mutation on Escherichia coli outer membrane proteins and lipopolysaccharide. J. Bacteriol. 188, 7186-7194. doi: 10.1128/jb.00571-06

Sklar, J. G., Wu, T., Gronenberg, L. S., Malinverni, J. C., Kahne, D., and Silhavy, T. J. (2007a). Lipoprotein SmpA is a component of the YaeT complex that assembles outer membrane proteins in Escherichia coli. Proc. Natl. Acad. Sci. U.S.A. 104, 6400-6405. doi: 10.1073/pnas.0701579104

Volokhina, E. B., Beckers, F., Tommassen, J., and Bos, M. P. (2009). The betabarrel outer membrane protein assembly complex of Neisseria meningitidis. J. Bacteriol. 191, 7074-7085. doi: 10.1128/jb.00737-09

Weirich, J., Brautigam, C., Muhlenkamp, M., Franz-Wachtel, M., Macek, B., Meuskens, I., et al. (2017). Identifying components required for OMP biogenesis as novel targets for antiinfective drugs. Virulence 8, 1170-1188. doi: $10.1080 / 21505594.2016 .1278333$

Sikdar, R., and Bernstein, H. D. (2019). Sequential translocation of polypeptides across the bacterial outer membrane through the trimeric autotransporter pathway. mBio 10:e01973-19.

Chauhan, N., Hatlem, D., Orwick-Rydmark, M., Schneider, K., Floetenmeyer, M., van Rossum, B., et al. (2019). Insights into the autotransport process of a trimeric autotransporter, Yersinia Adhesin A (YadA). Mol. Microbiol. 111, 844-862. doi: $10.1111 / \mathrm{mmi} .14195$

Tellez, R. Jr., and Misra, R. (2012). Substitutions in the BamA beta-barrel domain overcome the conditional lethal phenotype of a DeltabamB DeltabamE strain of Escherichia coli. J. Bacteriol. 194, 317-324. doi: 10.1128/jb.06192-11

Baba, T., Ara, T., Hasegawa, M., Takai, Y., Okumura, Y., Baba, M., et al. (2006). Construction of Escherichia coli K-12 in-frame, single-gene knockout mutants: the Keio collection. Mol. Syst. Biol. 2, 20060008.

Grosskinsky, U., Schutz, M., Fritz, M., Schmid, Y., Lamparter, M. C., Szczesny, P., et al. (2007). A conserved glycine residue of trimeric autotransporter domains plays a key role in Yersinia adhesin A autotransport. J. Bacteriol. 189, 90119019. doi: 10.1128/jb.00985-07

Conflict of Interest: The authors declare that the research was conducted in the absence of any commercial or financial relationships that could be construed as a potential conflict of interest.

Copyright () 2021 Rooke, Icke, Wells, Rossiter, Browning, Morris, Leo, Schütz, Autenrieth, Cunningham, Linke and Henderson. This is an open-access article distributed under the terms of the Creative Commons Attribution License (CC BY). The use, distribution or reproduction in other forums is permitted, provided the original author(s) and the copyright owner(s) are credited and that the original publication in this journal is cited, in accordance with accepted academic practice. No use, distribution or reproduction is permitted which does not comply with these terms. 